\title{
Recent Advances in Tuberculosis and Nontuberculous Mycobacteria Lung Disease
}

Jae Seuk Park, M.D., Ph.D.

Department of Internal Medicine, Dankook University College of Medicine, Cheonan, Korea

Tuberculosis (TB) is one of the largest health problems in the world today. And the incidence of nontuberculous mycobacteria (NTM) lung disease appears to be increasing worldwide. Recently, an automated, nucleic acid amplification assay for the rapid detection of both Mycobacterium tuberculosis and rifampin resistance was developed (Xpert MTB/RIF). And fixed-dose combinations of anti-TB drugs and linezolid have been introduced in the treatment of TB. And new NTM species, named Mycobacterium massiliense, which is very closely related to Mycobacterium abscessus was reported. In this review, these recent advances in the diagnosis and treatment of TB and clinical characteristics of $M$. massiliense lung disease are discussed.

Key Words: Tuberculosis; Nucleic Acid Amplification Techniques; Linezolid; Nontuberculous Mycobacteria

\section{Introduction}

Tuberculosis (TB) has been one of the world's deadliest diseases. In Korea, the incidence of TB has been decreased over decades, however, TB still remains a serious health problem considering that the estimated TB incidence was 100 (per 100,000 people) in 2011 ${ }^{1}$.

Rapid detection of Mycobacterium tuberculosis (MTB) and drug resistance to anti-TB drugs are critical for TB control $^{2}$. Recently, an automated, polymerase chain reaction (PCR) assay for the rapid detection of both MTB and rifampin resistance was developed (Xpert MTB/ RIF) $)^{3}$.

TB patients should take many drugs over 6 months for the treatment of TB. World Health Organization (WHO) recommended fixed-dose combinations (FDCs)

Address for correspondence: Jae Seuk Park, M.D., Ph.D. Department of Internal Medicine, Dankook University College of Medicine, 119 Dandae-ro, Dongnam-gu, Cheonan 330-997, Korea

Phone: 82-41-550-3055, Fax: 82-41-556-3256

E-mail: jspark@dankook.ac.kr

Received: May 11, 2013

Revised: May 15, 2013

Accepted: May 20, 2013

(a) It is identical to the Creative Commons Attribution Non-Commercial License (http://creativecommons. org/licenses/by-nc/3.0/). for delivering anti-TB drugs owing to better patient compliance and lesser chances of developing drug resistance ${ }^{4}$.

Extensively drug-resistant (XDR) TB (defined as in vitro resistance to isoniazid and rifampin plus any fluoroquinolone and at least one of the injectable drugs: amikacin, capreomycin, or kanamycin) is extremely difficult to treat because few effective anti-TB drugs are left available. Recently, several studies suggested that long term use of linezolid is effective in the treatment of $\mathrm{XDR} \mathrm{TB}^{5}$.

The incidence of nontuberculous mycobacteria (NTM) lung disease appears to be increasing worldwide including Korea. Thus diagnosis and treatment of NTM lung disease are challenging to physicians. Mycobacterium abscessus is the second most common cause of NTM lung disease, following Mycobacterium avium-intracellulare complex in Korea ${ }^{6}$. However, M. abscessus lung disease is difficult to treat and the optimal therapeutic regimens have not been established ${ }^{7}$. Recently, $M$. abscessus complex was shown to comprise three closely related species: $M$. abscessus, Mycobacterium massiliense, and Mycobacterium bolletii. And, M. abscessus and $M$. massiliense are isolated in almost equal numbers among $M$. abscessus complex infections in 
Korea ${ }^{8}$.

In this review, these recent advances in the diagnosis and treatment of TB and clinical characteristics of $M$. massiliense lung disease are summarised. And clinical significances and applicability to clinical practice in Korea are discussed.

\section{Xpert MTB/RIF}

Traditional smear and culture-based detection of MTB and drug resistance are time consuming and show relatively low sensitivity.

Recently developed Xpert MTB/RIF detect simultaneously MTB and rifampin resistance within 2 hours $^{3}$. Briefly, after 15 minutes of incubation, the mixture of sputum and buffer is pipetted into a single-use Xpert MTB/RIF cartridge, which is then loaded into the Xpert MTB/RIF machine. Then the machine automatically amplifies the genomic DNA by PCR and identifies MTB specific gene and mutations in the $r p o B$ gene (associated with rifampin resistance). Because buffer renders MTB non-viable within 15 minutes of incubation, Xpert MTB/RIF may be performed in the absence of a biosafety cabinet. And automated system enables the assay to be performed with ease by a minimally trained technician $^{3}$.

In a multi-center study, Xpert MTB/RIF test detected $90.3 \%$ of culture-confirmed cases of TB. Xpert MTB/RIF test sensitivity was $76.9 \%$ in smear-negative, culturepositive patients and specificity was $99.0 \%$. Smear microscopy sensitivity for MTB was lower in human immunodeficiency virus (HIV)-positive patients compared to HIV-negative patients (44.6\% vs. $68.6 \%$, respectively). But, Xpert MTB/RIF test sensitivity for MTB was not significantly lower in HIV-positive patients compared to HIV-negative patients (82.4\% vs. $90.7 \%$, respectively) ${ }^{9}$.

Xpert MTB/RIF showed high sensitivity (98\%) and positive predictive value (95\%) for the diagnosis of rifampin resistance in settings with high prevalence of rifampin-resistant TB (30\%). But, in a setting with lower prevalence of rifampin resistance $(2 \%)$, positive predictive value of rifampin resistance was less than $50 \%{ }^{10}$.
Thus, positive results for rifampin resistance should be interpreted carefully considering prevalence of rifampin resistance and individual level risk factors for rifampin resistance.

These findings suggest that Xpert MTB/RIF is suitable for the detection of both MTB and rifampin resistance in underdeveloped countries where multidrug-resistant (MDR) TB and HIV infections are common and adequate laboratory services are not available ${ }^{11}$.

However, the utility of Xpert MTB/RIF may be limited in developed countries like Korea, where MDR TB and HIV infections are rare and specialized laboratory services are available for detection of MTB and rifampin resistance by traditional PCR method ${ }^{12}$. However, Xpert MTB/RIF may benefit in circumstances where rapid detection of MTB and rifampin resistance is critical in the management of TB suspected patients.

\section{FDCs of Anti-TB Drugs}

Because TB patient should take three or four different drugs simultaneously for at least 6 months, ensuring adherence to treatment and avoiding inappropriate drug regimen are important in the treatment of $\mathrm{TB}$.

FDC is a formulation including two or more fixed doses of active drugs in a single dosage form.

The advantage of using FDCs in the treatment of TB is that patients have to take considerably fewer pills, thus making treatment easier, aiding adherence. Furthermore, because all drugs are provided in the same tablet, FDCs decrease the risk of developing drug resistance attributable to selective drug intake by the patient and prescription error ${ }^{13}$.

During the 1980s, substandard quality of FDCs and relatively poor bio-availability of rifampin were matters of concern ${ }^{14}$. However, current FDCs of anti-TB drugs are fully bio-equivalent to single-drug reference products, with stable efficacy ${ }^{15}$. And, several studies have shown that various combination of FDCs of first line anti-TB drugs are generally well tolerated and efficient similar to those of separate-drug regimens ${ }^{16}$.

Recently, a randomized controlled trial, conducted to 
evaluate the efficacy and safety of 4-drug (rifampin, isoniazid, pyrazinamide, ethambutol) FDC, also showed that 4-drug FDC regimen had an efficacy similar to that of a separate-drugs regimen ${ }^{17}$. Treatment-related adverse events were similar in both regimens. After resolution of adverse events, most patient in the FDC regimen moved to separate-drugs regimen because drugs in FDCs cannot be separated and it is difficult to identify the drug within the combination that is potentially responsible for an adverse event. Thus, patients treated with FDCs were more likely to be removed from the trial drugs than those treated with separate-drugs regimen.

Recently, 4-drug (rifampin, isoniazid, pyrazinamide, ethambutol) FDC was marketed in Korea (Tubes, BCworld Pharm Co. Ltd.). And Korea Centers for Disease Control and Prevention (KCDC) as well as WHO, recommended FDC for delivering anti-TB therapy ${ }^{4}$. However, stocks of single-drug tablets also should be made available for patients who develop adverse reactions to FDCs.

\section{Linezolid}

Linezolid (Zyvox), a recently developed oxazolidinone antibiotics, is used for the treatment of serious infections caused by gram-positive bacteria that are resistant to several other antibiotics like vancomycin ${ }^{18}$.

Several retrospective studies suggested that long term use of linezolid may be effective in treating MDR or $\mathrm{XDR} \mathrm{TB}^{5}$. In these studies, however, serious adverse events have been observed like neuropathies (e.g., peripheral and optic neuropathies) and myelosuppression.

Recently, a prospective, randomized trial was conducted to evaluate the effect and adverse events of linezolid in patients with chronic XDR TB who failed to show culture conversion with all other available chemotherapeutic options ${ }^{19}$. Patients were randomly assigned to linezolid therapy that started immediately or after 2 months, at a dose of $600 \mathrm{mg} /$ day, without a change in their background regimen. After confirmed sputumsmear conversion or 4 months, patients underwent a second randomization to continue linezolid therapy at a dose of $600 \mathrm{mg} /$ day or $300 \mathrm{mg} /$ day for at least an additional 18 months, with careful toxicity monitoring.

By 4 months, immediate start group showed significantly higher culture conversion rate compared to delayed-start group ( $79 \%$ vs. 35\%, respectively) ( $\mathrm{p}=$ 0.001). Eighty-seven percent of study patients showed sputum culture conversion within 6 months after linezolid had been added. Eighty-two percent of study patients had clinically significant adverse events related to linezolid, including 3 patients who discontinued linezolid permanently. Patients who received $300 \mathrm{mg} /$ day after the second randomization had fewer adverse events (69\%) than those who continued taking 600 $\mathrm{mg} /$ day (88\%). Seventy-three percent of patients, who showed adverse event with $600 \mathrm{mg} /$ day regimen, subsequently changed to $300 \mathrm{mg} /$ day regimen due to adverse events.

Three patients (two in the $300 \mathrm{mg}$ group, and one in the $600 \mathrm{mg}$ group) failed to show culture conversion and 1 patient in the $600 \mathrm{mg}$ group became culture negative but turned positive again after 1 year of treatment (relapse). The minimal inhibitory concentration (MIC) of linezolid against initial isolates of MTB from XDR TB patients were lower than peak plasma concentration of $300 \mathrm{mg} /$ day group.

These findings show that linezolid is highly effective in the treatment of XDR TB. And, lower dose regimen $(300 \mathrm{mg} /$ day) shows similar anti-TB effect with fewer adverse events than higher dose regimen (600 mg/day). However, patients must be monitored carefully for adverse events during treatment period.

\section{M. massiliense Lung Disease}

M. abscessus is a rapidly growing mycobacterium (RGM) causing a wide spectrum of disease in humans, including lung, skin, and soft tissue disease. In Korea, $M$. abscessus is the second most common pathogen responsible for NTM lung disease, following $M$. avium-intracellulare complex ${ }^{6}$.

$M$. abscessus is resistant to most antibiotics except 
some parenteral agents (amikacin, cefoxitin, imipenem) and macrolides (clarithromycin, azithromycin). Although combination therapy of intravenous amikacin with cefoxitin or imipenem and an oral macrolide has been recommended by the American Thoracic Society/Infectious Diseases Society of America and many other experts $^{20}$, treatment response rates are not satisfactory ${ }^{21}$.

In 2004, new RGM species, named $M$. massiliense, which is very closely related to $M$. abscessus was reported $^{22}$. M. abscessus was shown to comprise three closely related species: $M$. abscessus, $M$. massiliense, and $M$. bolletii. In Korea, M. abscessus and M. massiliense are isolated in almost equal numbers among $M$. abscessus complex infections, whereas $M$. bolletii is rare $^{8}$.

Recently, a retrospective clinical study was performed to compare clinical features and treatment outcomes between patients with $M$. abscessus and $M$. massiliense lung disease who were treated as $M$. abscessus lung disease previously ${ }^{23}$.

The regimen for the treatment of $M$. abscessus lung disease was taking clarithromycin, ciprofloxacin, and doxycycline for at least 12 months after sputum culture conversion along with an initial 4-week course of amikacin and cefoxitin. The clinical and radiographic manifestations of disease caused by each species were similar. Drug susceptibility test showed that the resistance rate of $M$. abscessus and $M$. massiliense to clarithromycin ( $5 \%$ vs. $4 \%$ ), amikacin ( $5 \%$ vs. $8 \%$ ), cefoxitin ( $0 \%$ vs. $1 \%$ ) were similar too. However, the proportion of patients with sputum culture conversion and maintenance of negative sputum cultures during the follow-up period was significantly higher in patients with $M$. massiliense infection (88\%) than in those with $M$. abscessus infection $(25 \%)(\mathrm{p}<0.001)$. Inducible resistance to clarithromycin (MIC> $>32 \mathrm{mg} / \mathrm{mL}$ ) was found in all tested $M$. abscessus isolates, but in none of the $M$. massiliense isolates. These findings suggest that the poor response of $M$. abscessus lung disease to treatment is due to induction of resistance to clarithromycin, an essential drug for the treatment of $M$. abscessus lung disease, during treatment period.
Because treatment response rates to clarithromycinbased antibiotic therapy were significantly higher in patients with $M$. massiliense lung disease than in those with $M$. abscessus lung disease, differentiation of $M$. massiliense from $M$. abscessus is important when RGM is isolated from NTM lung disease patients.

\section{Acknowledgements}

The present research was conducted by the research fund of Dankook University in 2012.

\section{References}

1. Park YK, Park YS, Na KI, Cho EH, Shin SS, Kim HJ. Increased tuberculosis burden due to demographic transition in Korea from 2001 to 2010. Tuberc Respir Dis 2013;74:104-10.

2. Farmer P, Bayona J, Becerra M, Furin J, Henry C, Hiatt $\mathrm{H}$, et al. The dilemma of MDR-TB in the global era. Int J Tuberc Lung Dis 1998;2:869-76.

3. Boehme CC, Nabeta P, Hillemann D, Nicol MP, Shenai S, Krapp F, et al. Rapid molecular detection of tuberculosis and rifampin resistance. N Engl J Med 2010; 363:1005-15.

4. Treatment of tuberculosis: guidelines [Internet]. 4th ed. Geneva: World Health Organization Web; 2009 [cited 2013 May 1]. Available from: http://www. who.int/tb/ publications/tb_treatmentguidelines/en/index.html.

5. Anger HA, Dworkin F, Sharma S, Munsiff SS, Nilsen DM, Ahuja SD. Linezolid use for treatment of multidrug-resistant and extensively drug-resistant tuberculosis, New York City, 2000-06. J Antimicrob Chemother 2010;65:775-83.

6. Koh WJ, Kwon OJ, Jeon K, Kim TS, Lee KS, Park YK, et al. Clinical significance of nontuberculous mycobacteria isolated from respiratory specimens in Korea. Chest 2006;129:341-8.

7. Colombo RE, Olivier KN. Diagnosis and treatment of infections caused by rapidly growing mycobacteria. Semin Respir Crit Care Med 2008;29:577-88.

8. Kim HY, Kook Y, Yun YJ, Park CG, Lee NY, Shim TS, et al. Proportions of Mycobacterium massiliense and Mycobacterium bolletii strains among Korean Mycobacterium chelonae-Mycobacterium abscessus group isolates. J Clin Microbiol 2008;46:3384-90.

9. Boehme CC, Nicol MP, Nabeta P, Michael JS, Gotuzzo 
E, Tahirli R, et al. Feasibility, diagnostic accuracy, and effectiveness of decentralised use of the Xpert MTB/RIF test for diagnosis of tuberculosis and multidrug resistance: a multicentre implementation study. Lancet 2011; 377:1495-505.

10. Steingart KR, Sohn H, Schiller I, Kloda LA, Boehme CC, Pai M, et al. Xpert(R) MTB/RIF assay for pulmonary tuberculosis and rifampicin resistance in adults. Cochrane Database Syst Rev 2013;1:CD009593.

11. Reid MJ, Shah NS. Approaches to tuberculosis screening and diagnosis in people with HIV in resource-limited settings. Lancet Infect Dis 2009;9:173-84.

12. Kirwan DE, Cardenas MK, Gilman RH. Rapid implementation of new TB diagnostic tests: is it too soon for a global roll-out of Xpert MTB/RIF? Am J Trop Med Hyg 2012;87:197-201.

13. Blomberg B, Spinaci S, Fourie B, Laing R. The rationale for recommending fixed-dose combination tablets for treatment of tuberculosis. Bull World Health Organ 2001;79:61-8.

14. Laserson KF, Kenyon AS, Kenyon TA, Layloff T, Binkin NJ. Substandard tuberculosis drugs on the global market and their simple detection. Int J Tuberc Lung Dis 2001;5:448-54

15. Agrawal S, Singh I, Kaur KJ, Bhade SR, Kaul CL, Panchagnula R. Comparative bioavailability of rifampicin, isoniazid and pyrazinamide from a four drug fixed dose combination with separate formulations at the same dose levels. Int J Pharm 2004;276:41-9.

16. Acceptability, compliance, and adverse reactions when isoniazid, rifampin, and pyrazinamide are given as a combined formulation or separately during three-timesweekly antituberculosis chemotherapy. Hong Kong
Chest Service/British Medical Research Council. Am Rev Respir Dis 1989;140:1618-22.

17. Lienhardt C, Cook SV, Burgos M, Yorke-Edwards V, Rigouts L, Anyo G, et al. Efficacy and safety of a 4-drug fixed-dose combination regimen compared with separate drugs for treatment of pulmonary tuberculosis: the Study C randomized controlled trial. JAMA 2011;305: 1415-23.

18. Leach KL, Brickner SJ, Noe MC, Miller PF. Linezolid, the first oxazolidinone antibacterial agent. Ann N Y Acad Sci 2011;1222:49-54.

19. Lee M, Lee J, Carroll MW, Choi H, Min S, Song T, et al. Linezolid for treatment of chronic extensively drugresistant tuberculosis. N Engl J Med 2012;367:1508-18.

20. Griffith DE, Aksamit T, Brown-Elliott BA, Catanzaro A, Daley C, Gordin F, et al. An official ATS/IDSA statement: diagnosis, treatment, and prevention of nontuberculous mycobacterial diseases. Am J Respir Crit Care Med 2007;175:367-416.

21. Jeon K, Kwon OJ, Lee NY, Kim BJ, Kook YH, Lee SH, et al. Antibiotic treatment of Mycobacterium abscessus lung disease: a retrospective analysis of 65 patients. Am J Respir Crit Care Med 2009;180:896-902.

22. Adekambi T, Reynaud-Gaubert M, Greub G, Gevaudan MJ, La Scola B, Raoult D, et al. Amoebal coculture of "Mycobacterium massiliense" sp. nov. from the sputum of a patient with hemoptoic pneumonia. J Clin Microbiol 2004; 42:5493-501.

23. Koh WJ, Jeon K, Lee NY, Kim BJ, Kook YH, Lee SH, et al. Clinical significance of differentiation of Mycobacterium massiliense from Mycobacterium abscessus. Am J Respir Crit Care Med 2011;183:405-10. 